\title{
Erratum to: Investigation of ototoxicity of artesunate as add-on therapy in patients with metastatic or locally advanced breast cancer: new audiological results from a prospective, open, uncontrolled, monocentric phase I study
}

\author{
Miriam König ${ }^{1}$ Cornelia von Hagens ${ }^{2} \cdot$ Sebastian Hoth $^{1} \cdot$ Ingo Baumann $^{1}$ \\ Ingeborg Walter-Sack ${ }^{3} \cdot$ Lutz Edler $^{4} \cdot$ Serkan Sertel $^{1}$
}

Published online: 19 April 2016

(C) Springer-Verlag Berlin Heidelberg 2016

\section{Erratum to: Cancer Chemother Pharmacol \\ (2016) 77:413-427}

DOI 10.1007/s00280-016-2960-7

The author would like to correct the errors in the publication of the original article. The corrected details are given below for your reading.

The conclusion section should read as:

Our results show that the continuous intake of ART for 4 weeks in doses up to $200 \mathrm{mg}$ daily was well tolerated concerning neuro-audiological function at all three doses tested in patients with metastatic or locally advanced breast cancer. However, a temporary dose-limiting vertigo was observed, two patients experienced ongoing subclinical hearing loss and another one an ongoing tinnitus. Therefore, regular audiological assessments should be included in clinical studies investigating oral ART in the treatment of cancer patients.

The online version of the original article can be found under doi:10.1007/s00280-016-2960-7.

Serkan Sertel

serkan.sertel@med.uni-heidelberg.de

1 Department of Otolaryngology, Head and Neck Surgery,

University of Heidelberg, Im Neuenheimer Feld 400,

69120 Heidelberg, Germany

2 Naturopathy and Integrative Medicine, Department of Gynecological Endocrinology and Reproductive Medicine, University Women's Hospital, Heidelberg, Germany

3 Department of Clinical Pharmacology and Pharmacoepidemiology, University Hospital, Heidelberg, Germany

4 Department of Biostatistics, German Cancer Research Center (DKFZ), Heidelberg, Germany 